\title{
ADMISSIBILITY OF SEMIGROUP STRUCTURES ON CONTINUA
}

\author{
BY \\ R. J. KOCH AND A. D. WALlACE( ${ }^{(1)}$
}

The purpose of this note is to investigate the structure of certain compact connected semigroups $S$ which satisfy $S^{2}=S$. If $X$ is any continuum then a continuous associative multiplication may be introduced by

(i) $x y=x$ all $x, y \in X$

or

(ii) $x y=y$ all $x, y \in X$.

We shall give certain instances in which, due to the topological structure of $S$, the multiplication must be of this trivial kind. The central ideas of the paper spring in one way or another from dense connected sets. For example, $S$ being a continuum ( $S$ will always denote a Hausdorff topological semigroup, or mob), $S^{2}=S$ if and only if each dense ideal is connected. We will show, for example, (Theorem 6) that if $S$ is irreducible between its minimal ideal $K$ and some other set and if $S^{2}=S$ then $S$ must have a unit, $S$ is commutative, and $S / K$ (in the metric case) is an arc. In a related result (Theorem 5) it will be proved that maximal ideals are composants. Because of the relative novelty of the subject and the present lack of structure theorems we go into considerable detail in considering some examples.

We follow the terminology of $[1$, p. 14]. In particular $K$ denotes the minimal ideal of $S$, if such exists, otherwise $K=\square$, and $E$ denotes the set of idempotents. If $S$ is compact it is known (see e.g. [14]) that $K$ and $E$ are nonempty and that $K$ is completely simple. If $S$ is connected and $K$ is nonempty, then $K$ is connected. For $a \in S, J(a)=a \cup S a \cup a S \cup S a S$ (the ideal generated by $a$ ). A clan is a compact connected mob with unit. We recall a result of [8]: if $S$ is compact and $S^{2}=S$, then $S=S E S$. This is equivalent to: if $S$ is compact, $S^{2}=S$, and $M$ is a proper ideal of $S$ then there is an idempotent outside $M$.

We first state a topological extension $[19 ; 20]$ of a theorem of ReesSuschkewitsch [13].

THEOREM 1. Let $S$ have a compact kernel (=minimal ideal) $K$, and let $e \in K$. Form eSe $\times(e S \cap E) \times(S e \cap E)$ with the multiplication $(t, e, f)\left(t^{\prime}, e^{\prime}, f^{\prime}\right)$ $=\left(t e f^{\prime} t, e^{\prime}, f\right)$ and define $\phi: e S e \times(e S \cap E) \times(S e \cap E) \rightarrow K$ by $\phi(t, e, f)=$ fet. Then $e S e \times(e S \cap E) \times(S e \cap E)$ is a compact mob, and $\phi$ is a topological isomorphism

Presented to the Society, November 30, 1956; received by the editors December 6, 1956.

(1) The authors are grateful for support from the National Science Foundation and the United States Air Force of Scientific Research, Air Research and Development Command, Contract No. AF 18(603)-89. 
onto. Further, if $\eta: K \rightarrow K$ is defined by " $\eta(t)$ is the idempotent in the group containing $t$," then $r: S \rightarrow K$ defined by $r(x)=x \eta(e x)$ is a retraction of $S$ onto $K$.

Our main use of Theorem 1 will be in the form of the following corollary:

Corollary 1. Let $S$ be a continuum, and suppose $K$ is not the cartesian product of two nondegenerate continua. Then either $K$ is a group or the multiplication in $K$ is of type (i) or (ii).

Proof. From Theorem 1 we know $K$ is topologically isomorphic with $e S e \times(e S \cap E) \times(S e \cap E)$, and each of the factors is a continuum since $S$ is. Hence at least two of $e S e, e S \cap E$, and $S e \cap E$ must consist of single elements. If $e S \cap E$ and $S e \cap E$ are single elements (namely $e$ ), then $K$ is isomorphic with $e S e$, hence is a group [1]. If $e S e$ and $e S \cap E$ are both the element $e$, then the multiplication is of type (i). If $e S e$ and $S e \cap E$ are both $e$, then the multiplication is of type (ii).

The following theorem extends a result of Faucett [4, Theorem 1.4].

Theorem 2. Let $S$ be compact and connected. Then $S^{2}=S$ if and only if each dense left (right) ideal containing $K$ is connected (also, if and only if, each dense ideal is connected).

Proof. Suppose $S^{2}=S$ and let $L$ be a dense left ideal containing $K$. Then $S L=S(L \cup K)=\cup\{S a \mid a \in L\} \cup K$ and each $S a$ meets the connected set $K$, so $S L$ is connected. Now $S L \subset L \subset S$ and $(S L)^{*}=S^{2}=S$ (stars denote closure) so $L$ is connected. Conversely, suppose each dense left ideal containing $K$ is connected and $S^{2} \neq S$. Let $a \in S \backslash S^{2}$ and let $V$ be an open set about $a$ with $V^{*} \cap S^{2}=\square$. Then $S \backslash F(V)$ is a dense two-sided ideal and is not connected, a contradiction.

Lemma 1. Let $S$ be connected, let $K$ exist and let $S=E S$. Then each left ideal containing $K$ is connected.

Proof. Let $L$ be a left ideal containing $K$. Since $S=E S$ it follows that $L=S L$. Then $L=S L=S(L \cup K)=\bigcup\{S a \mid a \in L\} \cup K$ and each $S a$ meets the connected set $K$, so $L$ is connected.

We say that a continuum has property $\left(*_{n}\right)$ if each dense connected open subset omits at most $n$ points.

Lemma 2. Let $S$ be a continuum satisfying $\left(*_{2}\right)$, and with $S^{2}=S$. Then either $S$ has a left or right unit, or for some e, $f \in E, S=S e \cup S f=e S \cup f S$.

Proof. It is known that each maximal proper (left, right, 2 -sided) ideal is open and dense $[9$, p. 681-682]. Note that $S$ has at most two maximal proper ideals (m.p.i.): for if $M_{1}, M_{2}, M_{3}$ are m.p.i. then $M_{1} M_{2} M_{3} \subset M_{1} \cap M_{2} \cap M_{3}$. Now $M_{1} M_{2} M_{3}$ is dense and connected by continuity of multiplication, hence $M_{1} \cap M_{2} \cap M_{3}$ is dense. It is open since each of $M_{1}, M_{2}, M_{3}$ is open, and finally 
it is connected since it lies between a connected set and its closure. Hence $M_{1} \cap M_{2} \cap M_{3}$ is dense, connected, open, and omits at least three points.

CASE 1. $M$ is the unique m.p.i. Then $M$ omits at most two elements, and at least one of these is an idempotent, say $M \subset S \backslash e$. Now $S e$ and $e S$ may be assumed to be proper since otherwise $S$ would have a left or right unit. Hence $S e \subset L$, a maximal proper left ideal and $e S \subset R$, a maximal proper right ideal. Let $a \in S \backslash L$ and $b \in S \backslash R$. Now $R M L \subset R \cap M \cap L$ so $R \cap M \cap L$ is dense, connected, open and omits $e, a, b$. Hence $a=b, R=L$, and $L$ is an ideal. Since $M$ is unique, $e \in L \subset M$, a contradiction.

CASE 2. $M_{1}, M_{2}$ are distinct m.p.i. Then $M_{1}=S \backslash e, M_{2}=S \backslash f$ where $e, f \in E$. If $S e \cup S f$ is proper, then $S e \cup S f \subset L$, a maximal proper left ideal. Let $a \in S \backslash L$. Then $M_{1} M_{2} L \subset M_{1} \cap M_{2} \cap L$ and this omits the three points $e, a, f$. We conclude that $S e \cup S f=S=e S \cup f S$, and the proof is complete.

In what follows, we use the Alexander-Kolmogoroff cohomology groups based on some fixed but arbitrary nonzero Abelian group for coefficients.

Lemma 3. Let $S$ be a continuum with either left or right unit, or with $S=E_{1} S$ $=S E_{2}$ where $E_{1} \cup E_{2} \subset E$ and one of $E_{1}, E_{2}$ is finite. Then $H^{1}(S, K)=0$.

Proof. Note that $H^{1}(S, K)$ is isomorphic with $H^{1}(S / K)$ where $S / K$ is the Rees quotient of $S$ by $K[16]$. Since $S / K$ has a zero and satisfies the hypotheses of the lemma, it suffices to consider the case when $S$ has a zero. It now remains to show $H^{1}(S)=0$. If $S$ has either a left or right unit, the result is known [16]. Suppose $E_{2}$ is finite and assume inductively that $H^{1}(S)=0$ if card $E_{2} \leqq k$. Suppose $S=E_{1} S=S E_{2}$ with $E_{2}=\left\{e_{1}, e_{2}, \cdots, e_{k}, f\right\}$, and let $S_{1}=\bigcup_{i=1}^{k} S e_{i}$. Consider the Mayer-Vietoris exact sequence [3] (using reduced groups in dimension 0 ):

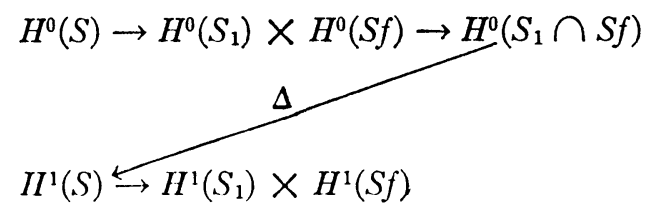

Now $S_{1}$ and $S f$ are connected since $S$ has a zero, so $H^{0}\left(S_{1}\right)=0$ and $H^{0}(S f)=0$. Also $H^{1}\left(S_{1}\right)=0$ by the induction hypothesis, and it is known (since $S$ has a zero) that $H^{1}(S f)=0[16]$. It follows that $\Delta$ is an isomorphism onto, and it remains to show that $H^{0}\left(S_{1} \cap S f\right)=0$, i.e. that $S_{1} \cap S f$ is connected. Since $S$ has a zero and $S=E_{1} S$, this is immediate from Lemma 1, and the proof is complete.

We say that a continuum $X$ has property $\mathrm{N}$ if for any proper subcontinuum $P \subset X, H^{1}(X, P) \neq 0$.

TheOREM 3. Let $S$ be a continuum satisfying $\left(*_{2}\right)$, having property $\mathrm{N}$, and with $S^{2}=S$. Then $S=K$, and either $S$ is a group or the multiplication is of type (i) or (ii). 
Proof. We conclude from Lemmas 2 and 3 that $H^{1}(S, K)=0$. Hence if $K<S$, then $S$ fails to have property $\mathrm{N}$. Therefore $S=K$, and since $S$ cannot be the cartesian product of two nondegenerate continua by $\left(*_{2}\right)$, the conclusion follows from Corollary 1.

Some examples covered by Theorem 3 are: $S^{1}$, two tangent $S^{1}$ 's, $S^{1}$ together with a diameter, two disjoint $S^{1}$ 's connected by a line segment, and variations of these in which arcs are replaced by "long" arcs (based on the ordinal numbers filled in by arcs). It is likely that the only mob satisfying the hypotheses of Theorem 3, and which admits a group structure is $S^{1}$. At this writing it is unknown whether Theorem 3 holds with $\left(*_{2}\right)$ replaced by $\left(*_{n}\right)$, and in particular by $\left(*_{3}\right)$.

Definition. Let $X$ be a continuum, $p \in X$, and define $T(p)$ to be the intersection of the sets $(X \backslash A)^{*}$ where $A$ runs through all continua in $X$ not containing $p$. As stated in [7], $T(p)$ is a continuum if $X$ is metric. It can be seen that this holds without the assumption that $X$ is metric.

THEOREM 4. If $S$ is a clan, if $I$ is an open or closed ideal, and if $p \in S$, then $T(p) \cap I \neq \square$ implies $p \in I$.

Proof. Let $I$ be closed, $p \in S \backslash I, x \in T(p) \cap I$. Let $U$ be an open set about $p$ with $U^{*} \cap I=\square$. Now $J_{0}\left(S \backslash U^{*}\right)$, the largest ideal of $S$ contained in $S \backslash U^{*}$, is an open connected ideal [9] containing $I$. Then $J_{0}\left(S \backslash U^{*}\right)^{*}$ is a continuum containing $x$ in its interior which does not contain $p$. Hence $x \in S \backslash T(p)$, a contradiction. Now let $I$ be open, $p \notin I, x \in T(p) \cap I$. Let $U$ be an open set about $x$ with $U^{*} \subset I$. Note that $S U^{*} S$ is an ideal and hence is connected since $S$ has a unit. Then $S U^{*} S$ is a continuum containing $x$ in its interior which does not contain $p$. Hence $x \in S \backslash T(p)$, a contradiction.

Corollary. If $S$ is a clan and if $p \in H_{u}$ (the maximal subgroup containing the unit), then $T(p) \subset H_{u}$.

Proof. Note that $S \backslash H_{u}$ is a maximal proper ideal. If $x \in T(p) \backslash H_{u}$, then $S \times S$ is a closed ideal containing $x$, so by the theorem, $p \in S \times S$. But $p \in H_{u}$ implies $H_{u} \subset S \times S \subset S \backslash H_{u}$, a contradiction.

EXAMPLE 1. Let $A$ be the graph of $\sin x^{-1}, 0<x \leqq 1$ together with the limit line, let $B=\{(2-x, y) \mid(x, y) \in A\}$, and let $X=A \cup B$. Denote the limit lines by $L$ and $R$. We show that if $X$ admits the structure of a mob $S$ equal to its square then the multiplication must be of type (i) or (ii). Suppose $S^{2}=S, K<S$ and let $J$ be a maximal proper ideal. Then there is an idempotent $e$ outside $J$, and since $J$ is open, dense, and connected by Theorem 2, we have $e \in L \cup R$. We show now that if $K \cap L \neq \square$, then $K \cup L$ is an ideal. If $L \subset K$ then the claim is clear. Hence we may assume $K \subset L$. Now $S L$ $=L L \cup[S \backslash(L \cup R)] \cdot L \cup R L$ and each of these sets is arcwise connected and meets $K \subset L$. Hence each set is contained in $L$ since no arcwise connected set can meet both $L$ and its complement. In a similar way $L \cup K(=L)$ is a right 
ideal, so $L \cup K$ is an ideal. In a similar way we can show that if $K \cap R \neq \square$ then $K \cup R$ is an ideal. Since $K$ is proper, either $K \cap L=\square$ or $K \cap R=\square$. Also either (1) $K \cap L=\square$ and $e \in L$ or (2) $K \cap R=\square$ and $e \in R$. Suppose (1) holds. Let $f$ be any idempotent in $L$. Then $f S f$ is a continuum containing $f$ and a point of $K$ (which is outside $L$ ). Hence $L \subset f S f$, so $f$ is a unit for $L$. It follows that $e$ is the unique idempotent of $L$. Since $L$ is arcwise connected, so is $L^{2}$, and since $e \in L$ we conclude $L^{2} \subset L$. It follows that $L$ is a group, but $L$ is not homogeneous, a contradiction. Under (2) we reach a similar contradiction. Hence $K=S$ and the conclusion follows from the Corollary to Theorem 1 .

EXAmple 2. [11, p. 93]. Let $p$ be the origin of the plane, $a=(1,0)$, $b=(-1,0), b_{n}=(-1,-1 / n), c_{n}=((n+1) / n,-1 / n), e_{n}=((n+1) / n, 1 / n)$. Let $L$ be the line segment from $b$ to $a$, let $x_{n}$ be the arc from $b_{n}$ to $p, x_{n}$ $=\left[b_{n}, c_{n}\right] \cup\left[c_{n}, e_{n}\right] \cup\left[e_{n}, p\right]$. Let $S=L \cup x_{1} \cup x_{2} \cup \ldots$. We show that $S$ does not admit the structure of a mob with unit. Suppose the contrary. We first show $K \cap L=\square$. Suppose not; then if $x$ is a point of $L$ to the right of $p$, we have $T(x)=L$, so $T(x) \cap K \neq \square$ and $x \in K$. Note that no subcontinuum of $S$ is a cartesian product of two nondegenerate subcontinua, so by the Corollary to Theorem 1 we may assume $K$ consists of left zeroes, i.e. each element of $K$ is a minimal right ideal. Let $V$ be an open set about $x$, let $R(V)$ be the largest right ideal of $S$ contained in $V$. Then $R(V)$ is open [9] and $R(V) \cup K$ is connected (by the dual to Lemma 1 ). We conclude that $K$ contains all but a finite number of line segments converging to $[p, a]$, (the segment from $p$ to $a)$. Now the unit $u$ is a non cutpoint, and we distinguish two cases. Suppose $u \in S \backslash L$; pick sequences $\left\{w_{n}\right\}$ and $\left\{z_{n}\right\}$ in $k$ with $\left\{z_{n}\right\} \rightarrow p$ and $\left\{w_{n}\right\} \rightarrow x$. Then $u z_{n}=z_{n}, p z_{n}=p$ for each positive integer $n$ so by arcwise connectedness there is a sequence $\left\{t_{n}\right\}$ in $[p, u]$ with $t_{n} z_{n}=w_{n}$. Now $\left\{t_{n}\right\}$ clusters at $t_{0} \in[p, u]$ so $\left\{t_{n} z_{n}\right\}=\left\{w_{n}\right\}$ clusters at $t_{0} p$ and converges to $x$, hence $t_{0} p=x$. We show next that $[p, u]$ is a mob, which will furnish a contradiction. Let $q$ be that point of $K$ in $[p, u]$ which is closest to $u$. By a result of Faucett [4], [q,u] is a mob. Let $r$ be that point of $[p, q]$ which is closest to $q$ and satisfies $p \in[u, q] r$, so that $[u, r]^{2}=[u, p]$. Using the fact that $[q, r]$ consists of left zeroes we see that $[u, r]^{3}=[u, r]^{2}$. Hence $\cup\left\{[u, r]^{n} \mid n \geqq 1\right\}=[p, u]$ is a mob. Hence we may suppose $u \in L$; let $\left\{x_{n}\right\}$ be a sequence of endpoints converging to $u$. Then $u x_{n}=x_{n}$ and $p x_{n}=p$ for each $n$, so that, by arcwise connectedness, there is a sequence $\left\{t_{n}\right\}$ in $[u, p]$ with $t_{n} x_{n}=w_{n}$. Then $\left\{t_{n}\right\}$ clusters at $t_{0} \in[u, p]$ so $\left\{t_{n} x_{n}\right\}=\left\{w_{n}\right\}$ clusters at $t_{0} u$ and converges to $x$, hence $t_{0}=x$, a contradiction. Thus $K \cap L=\square$ so that $K$ is an interval. We shrink $K$ to a point to get the Rees quotient [13], and now have a zero (0). Let $y$ denote the endpoint of the line on which 0 lies. Then $[y, p)$ is an open set about 0 , so $J=J_{0}([y, p))$, the largest ideal of $S$ contained in $[a, p)$, is an open ideal with $J^{*} \cap L=p$. Hence $T(x) \cap J^{*} \neq \square$, so that $x \in J^{*}$, the contradiction which completes the argument.

Recall that $A$ is an isolated arc in the space $X$ if $A$ is an arc and $A \cap(X-A) *$ is one endpoint of $A$. 
RemArk. Let $X$ be a space containing an isolated arc. Then $X$ admits the structure of a mob $S$ with left unit and with $S \neq K$.

Proof. Let $A$ be an isolated arc in $X, B=(X-A)^{*}, p=A \cap B$. We define the multiplication in $B$ to be of type (ii). Let the multiplication in $A$ be that of the usual unit interval with $p$ playing the role of zero. Finally, for $x \in A, y \in B$, let $x y=y, y x=p$.

The construction can perhaps be seen more easily in the following way: using the multiplications in $A$ and $B$ given above, let $A \times B$ have coordinatewise multiplication. Then $A \cup B$ is homeomorphic with the mob $(A \times p)$ $\cup(p \times B)$.

We now take up some results of a preliminary nature involving a mob $S$ which is a continuum irreducible between two subsets $A$ and $B$ and with $S^{2}=S$. Recall that $S$ is irreducible between $A$ and $B$ if for any subcontinuum $C$ with $C \cap A \neq \square \neq C \cap B$ we have $C=S$. For a compact Hausdorff space $X$, $c d X \leqq n$ iff the natural map $H^{n}(X) \rightarrow H^{n}(A)$ is onto for each closed set $A \subset X$ [2]. (Here $c d X$ is based on a fixed, but arbitrary, coefficient group.)

THEOREM 5. Let $S$ be a continuum with neither left nor right unit such that $S^{2}=S$ and $S$ is irreducible between two subsets $A$ and $B$. Then

(1) $A \subset H_{e}, B \subset H_{f}$ for some $e, f \in E \backslash K$, and $S=e S e \cup f S f$.

(2) There are exactly two maximal proper ideals, $S \backslash H_{e}$ and $S \backslash H_{f}$.

(3) $I H_{e}$ and $H_{f}$ are connected.

(4) $S$ has exactly three composants, $S, S \backslash H_{e}$ and $S \backslash H_{f}$.

(5) If $c d S=1$, then $A=H_{e}=e, B=H_{f}=f$.

Proof of (1). Let $p \in A, q \in B$; since $S^{2}=S, p \in x S$ and $q \in S y$ for suitable $x, y \in S$. Hence by the irreducibility $S=x S \cup S y$ and $x S=x^{2} S \cup x S y$, so that $S=x^{2} S \cup x S y \cup S y=\cdots=x^{n} S \cup S y$ for each positive integer $n$. Therefore $S=e^{\prime} S \cup S y$ where $e^{\prime} \in E \cap \Gamma(x)$ [8, Theorem 2]. By a similar argument $S=e^{\prime} S \cup S f^{\prime}$ where $f^{\prime} \in \Gamma(y)$. If $p \notin e^{\prime} S$ then $p \in S f^{\prime} \subset S y$ so that $p, q \in S y$ and $S y=S$; it follows that $S$ has a right unit. Hence $p \in e^{\prime} S$ and similarly $q \in S f^{\prime}$, $p \in S p, q \in q S$, and $S=S p \cup q S$. Now $S=S e \cup f S$ for suitable idempotents $e, f$, and $p \in S e, q \in f S, p \in p S, q \in S q$. Hence $S=p S \cup S q$, so $S=e S \cup S f$. If $p \notin e S$, then $p, q \in S f, S f=S$, and $f$ is a right unit for $S$. Hence $p \in e S$ and similarly $q \in S f$. Hence $p \in e S \cap S e=e S e$ and $q \in f S \cap S f=f S f$, so by the irreducibility $S=e S e \cup f S f$. Since $e \in p S \cap S p$ it follows that $p \in H_{e}$ and in a similar way $q \in H_{f}$.

We now show $e, f \in S \backslash K$. If $e \in K$, then either $(e S e)^{0}=\square$ or $e S e=K$ [15, Theorem 4]. The first of these is impossible since $e \notin f S f$. But if $e S e=K$, then $K$ is a group [14], hence $K \subset f S f$. Then $e \in K \subset f S f$, the contradiction which completes the proof of (1).

Proof of (2). Note that any maximal proper ideal $M$ misses either $A$ or $B$, otherwise for $a \in A \cap M, b \in B \cap M$ we have $J(a) \cup J(b) \subset M<S$. But by Lemma $1, J(a) \cup J(b)$ is a continuum which meets both $A$ and $B$, so that $S$ 
is not irreducible. It follows that there are at most two maximal proper ideals and we now show that $S \backslash H_{e}$ and $S \backslash H_{f}$ are distinct maximal proper ideals. The distinctness is clear and it suffices to show that $S \backslash H_{e}$ is an ideal. If not, then $S \backslash H_{e}$ fails, say, to be a left ideal, so for some $x \in S$ and $y \in S \backslash H_{e}, x y \in H_{e}$. We conclude that $e \in S y$; further since $S=e S e \cup f S f$ and $S$ has neither a left nor a right unit it follows that $y \in e S e$. Hence $e=z y$ for some $z \in S$ and we may assume $z, y \in e S e$. Now $(y z)(y z)=y z$. So by the complete simplicity of $S e S /\left(S e S \cap J_{0}(S \backslash e)\right)$ [6, Corollary 4] either $y z=e$ or $y z \in J_{0}(S \backslash e)$. If $y z \in J_{0}(S \backslash e)$ then $e=(z y)=z(y z) y \in J_{0}(S \backslash e)$, a contradiction. Hence $y z=e$ and $y \in H_{e}$. This contradiction completes the proof.

Proof of (3). We establish the following more general result. The arguments used are similar to those in [17, Lemma 3]. Let $S$ be a continuum, $S=E S E, K \neq S, S$ irreducible about $A$, and let $J$ be a maximal proper ideal. Then $A^{*}$ meets each component of $S \backslash J$.

Proof. Let $C$ be a component of $S \backslash J$, suppose $A^{*} \cap C=\square$, and let $U$ be an open set about $C$ with $F(U) \cap(S \backslash J)=\square$, and with $A^{*} \cap U^{*}=\square$. Then $F=F(U) \subset J$ so $S F S \subset J$. Since $J$ is open, there is an open set $V$ about $F$ with $V \cap K=\square$ and $S V^{*} S \subset J$. Now $S \backslash F=\left(S \backslash U^{*}\right) \cup U$ so $S \backslash S V^{*} S$ $=\left[S \backslash\left(U^{*} \cup S V^{*} S\right)\right] \cup\left[U \backslash S V^{*} S\right]$ and these are separated. (Note that $F \subset S V^{*} S$.) If $W=U \backslash S V^{*} S$ then $S \backslash W$ is connected, $W \subset U$ and $C \subset W$. Hence $A \cap W=\square$ and $(S \backslash W)^{*}$ is a proper continuum containing $A$, violating the irreducibility.

Proof of (4). We show that $C_{e}$, the composant of $S$ containing $e$, is $S \backslash H_{f}$. Let $x \in S \backslash H_{f}$; then $J(x) \cup K \cup e S e$ is a proper continuum containing $e$ and $x$ so that $x \in C_{e}$. Now let $x \in C_{e}$, so that $x, e \in P$, a proper subcontinuum of $S$. Suppose $x \in H_{f}$; then $H_{e} \cup P \cup H_{f}$ is a continuum containing $p$, $q$, so $H_{e} \cup P \cup H_{f}$ $=S$. But $H_{e}$ has no inner points since maximal proper ideals are dense, and similarly for $H_{f}$. Hence $P=S$, a contradiction.

Proof of (5). We first form the Rees quotient $S / K$, i.e. we shrink $K$ to a point. If $f: S \rightarrow S / K$ is the natural map, then $S / K$ is a continuum irreducible between $f(A)$ and $f(B)$ and has a zero. Hence we may assume $S$ has a zero. If $H_{e} \neq e$, then $H_{e}$ is a compact connected one-dimensional group, so $H^{1}\left(H_{e}\right)$ $\neq 0$ and $H^{1}(e S e) \neq 0$ (real coefficients). But $e S e$ is now a clan with zero, and it is known [16, p. 48] that $H^{n}(e S e)=0$ (all $\left.n \geqq 0\right)$, a contradiction.

Recall that a subset $C$ of a continuum $X$ is a $C$-set if for any subcontinuum $A$ of $X$ with $A \cap C \neq \square$ we have either $A \subset C$ or $C \subset A$.

Theorem 6. Let $S$ be a continuum such that $S^{2}=S$ and such that $S$ is irreducible between $K$ and $A$, where $A \subset S$. Then

$S$ has a unit, $S$ is commutative, and $S / K$ is irreducibly connected between zero and unit.

The proof will proceed in several short steps, as follows:

(1) $S$ has a unit $u, A \subset H_{u}$, and $H_{u}$ is the set of all $x$ such that $S$ is irreducible between $K$ and $x$. 
(2) $K$ is a group, each closed left ideal is principal, $x S=S x$ for each $x \in S$, and $x, y \in S$ imply $S x \subset S y$ or $S y \subset S x$.

(3) The subcontinua of $S$ meeting both $K$ and $S \backslash K$ coincide with the principal ideals $\neq K, I(p)$ is open for each $p \in S \backslash K$, and each set $J_{a}, a \in S$, is a $C$-set in $S a$.

(4) $J_{a}=a$ for each $a \in S \backslash K$, so $S / K$ is irreducibly connected between zero and unit.

(5) $S$ is commutative.

Proof of (1). Let $a \in A$; since $S^{2}=S, a \in S x$ for some $x \in S$. Now $S x$ is a continuum which meets $K$ and contains $a$ so that $S=S x$. Hence $S=S e$ where $e \in \Gamma(x) \cap E$, and $e$ is a right unit. Dually $S$ has a left unit so that $S$ has a unit. Note that by the result stated in the proof of Theorem 5, Part (3), $H_{u}$ is connected. We show next that $H_{u}$ is the set of all $x$ such that $S$ is irreducible between $K$ and $x$. Suppose $S$ is irreducible between $K$ and $x$; then $S x=S$ so $x \in H_{u}$. Conversely if $x \in H_{u}$, then let $P$ be a continuum with $x \in P$ and $P \cap K \neq \square$. If $a \in A$, then $a x^{-1} P=S$, and by [18, Theorem 1] we conclude that $P=S$.

The following lemma will be useful in the remaining proofs.

Lemma 4. Let $S$ satisfy the hypotheses above, and let $L \subset S$ be a closed left ideal containing $K$. Then $x \in S-L$ implies $S x \supset L$.

Proof. Let $U$ be an open set about $x$ with $U^{*} \cap L=\square$. Let $P=L_{0}\left(S \backslash U^{*}\right)$, the largest left ideal of $S$ contained in $S \backslash U^{*}$. Since $S$ has a unit by (1), $P$ is connected by Lemma 1 , and $P$ is known to be open [9]. Since $K \subset P$, it follows from $\left[10\right.$, p. 134] that $\left(S \backslash P^{*}\right)^{*}$ is a continuum (which misses $L$ ). Hence $S x \cup\left(S \backslash P^{*}\right)^{*}$ is a continuum which meets $K$ and contains $u$, so $S x \cup\left(S \backslash P^{*}\right)^{*}=S$ and $L \subset S x$.

Proof of (2). We show first that $K$ is a group. If we take $L=K$ in Lemma 4, and let $y \in F(K)$, it follows from continuity of multiplication that $S y=K=y S$. Hence $K$ is a minimal left and right ideal, so $K$ is a group [1]. Since $K$ is a group, each left ideal must contain $K$, and the hypotheses of Lemma 4 can be relaxed accordingly. By an argument similar to that just given for $K$, we see that each closed left ideal is principal. We show next that $S x=x S$ for each $x \in S$. To show that $S x \subset x S$ let $y \in S x$ and suppose $y \in S \backslash x S$. By the dual of Lemma $4, x S \subset y S \subset S x S$. But $x S$ is maximal among the principal right ideals generated by elements of $S x S[8$, p. 399], so $x S=y S$ and $y \in x S$, a contradiction. In a similar way, $x S \subset S x$, so $S x=x S$. Finally let $x, y \in S$ and suppose $S x \nsubseteq S y$. Note that $x \in S \backslash S y$ so by Lemma 4, SyCSx and (2) is complete.

Proof of (3). Let $P$ be a continuum of $S$ which meets both $K$ and $S \backslash K$. Then $P \subset S P$, and since each closed left ideal is principal, $S P=S x$ for some $x \in S$. Also $x \in S P$, so $x \in S p$ for some $p \in P$, and $S P=S x=S p$ (note that $p \in S \backslash K)$. Hence $P \subset S p$, and we now show $S p \subset P$. Let $J_{p}=\{x \mid S x S=S p S\}$, 
and let $I(p)=J(p) \backslash J_{p}$. It is clear that $I(p)$ is an ideal of $S$, and we first show $I(p)$ is open. Let $x \in I(p)$; by (3), $S x S=S x=x S$, so $S x<S p$. Let $V$ be an open set about $S x$ with $p \in S \backslash V^{*}$. Let $L$ be the largest left ideal of $S$ contained in $V$; then $L$ is open [9] and $p \in S \backslash L^{*}$. Hence by Lemma $4, x \in L \subset L^{*}$ $C S p$, so $I(p)$ is open. Now let $y \in I(p)$, and let $W$ be an open set about $y$ with $W \subset I(p)$ and $W^{*} \cap J_{p}=\square$. Then $\left(S \backslash S W^{*}\right)^{*} \cup P$ is a continuum which meets both $A$ and $K$, so that $\left(S \backslash S W^{*}\right)^{*}=S$ and it follows that $x \in P$. Hence $I(p) \subset P$ and $S p=J(p)=I(p) * \subset$.

Finally we show that each $J_{a}, a \in S$, is a $C$-set in $S a$. Let $Q$ be a subcontinuum of $S a S$ with $Q \cap J_{a} \neq \square \neq Q \cap I(a)$, and let $x \in Q \cap I(a)$. Then $S x S \cup Q$ is an ideal by the above results, hence $S x \cup Q=J(a)$. Since $S x \subset I(a) \subset S \backslash J_{a}$, $J_{a} \subset Q$ and $J_{a}$ is a $C$-set. It is known $[15$, p. 639] that a $C$-set is connected and has no inner point.

Proof of (4). Let $a \in S$ and we will show $J_{a}=a$. We distinguish two cases, according as $J_{a}$ is a group or not. We note that $J_{a}$ is a group if and only if $J_{a} \cap E \neq \square$. This follows from the complete simplicity of $J(a) / I(a)[6]$ and the fact that $S x=x S$ for each $x \in S$. Suppose $J_{a}$ is not a group; let $S e$ be a minimal member of $[S f \mid a \in S f, f \in E]$. Then in the sense of the relation $a \leqq b \leftrightarrow a \in S b, e$ is the smallest idempotent above $a$ (the uniqueness is a consequence of part (2)). It follows that $V=S e \backslash S a$ is an open set relative to $S e$ containing $e$ such that $V \cap E=\{e\}$. By a theorem of Mostert and Shields [12] an $\operatorname{arc} A$ can be started at $e$ such that $A \subset S e$ and $A \cap J_{e}=\{e\}$. Since $J_{e}$ is a $C$-set in $S e$, we conclude that $J_{e}=e$. Now $S b=[x \mid x \leqq b]$ is closed for each $b \in S$. Hence [21] there is a smallest ( $\leqq$ ) element $z$ with $z a \in J_{a}$. We now show that $J_{z} \cdot a=J_{a}$. If $y \in J_{z}$ and $y a \in I(a)$, then $z a \in S z a=S y a \subset I(a)$, a contradiction. Now let $b \in J_{a}$ and suppose that $b \in J_{z} \cdot a$. Note that $b \in I(z) a$, otherwise (using the minimality of $z$ ) $z a \in z S b \subset S I(z) \cdot a \subset I(a)$, contrary to fact. Hence $b \notin\left[I(z) \cup J_{z}\right] \cdot a=J(z) \cdot a=S z a=S a$, a contradiction. Hence $J_{z} \cdot a=J_{a}$, so $a=t a$ for some $t \in J_{2}$, and $a=t a=t^{2} a=\cdots=t^{n} a$ for each positive integer $n$. Therefore $a=f a$ for $f \in E \cap \Gamma(t)$, and $a \in f S$. We conclude from the minimality of $e$ that $f=e, t \in H_{e}$, and $J_{z}=H_{e}=\{e\}$. Hence $J_{a}=J_{z} \cdot a=e a=a$.

Now suppose $J_{a}$ is a group, $J_{a}=J_{e}=H e$ with $e^{2}=e$. Suppose $J_{e} \neq e$, and consider $S e$. Since $H_{e}$ is not a $D$-chain in $S e$, [4, Theorem 2.2], there is an open set $V_{1}$ (relative to $S e$ ) about $H_{e}$ such that $V_{1}$ contains no cutpoint of $S e$. Let $V$ be an open set (relative to $S_{e}$ ) about $H_{e}$ with $V^{*} \subset V_{1}$. If $V \cap(E \backslash e)=\square$, then an arc (in $S e$ ) can be started at $e$ and as in the first part of the proof we conclude $J_{e}=e$. Hence we may assume $V \cap(E \backslash e) \neq \square$. We show next that $V^{*} \cap E$ is a compact order dense ( $\leqq$ )-chain. It is clearly a compact chain. To show $V^{*} \cap E$ is order-dense, suppose $f, g \in V^{*} \cap E$ and $g$ "covers" $f$, that is, $g$ is the least element with $f \in S g$ and $g \neq f$. Then there is $W$ open (relative to $S g$ ) about $g$ with $W \subset V$ and $f \in S \backslash W$. Now $W \cap(E \backslash g)=\square$, so an arc $A$ can be started in $S g$, with $A \cap J_{g}=\{g\}$. Since $J_{g}$ is a $C$-set we conclude that $J_{\theta}=\{g\}$, hence $g$ is a cutpoint of $S$ since $I(g)$ is open. But $V$ contains no cut- 
points of $S$, a contradiction. It is clear that the topology induced by $\leqq$ on $E$ coincides with the relative topology of $E$. Hence $V^{*} \cap E$ is an order dense compact chain and it follows that $V^{*} \cap E$ is a continuum. Since $J_{e}$ is a $C$-set and $V^{*} \cap E \cap J_{e}=\{e\}$, we conclude that $J_{e}=\{e\}$. Therefore $J_{a}=\{a\}$ for each $a \in S \backslash K$, and each $a \in S \backslash K$ is a cutpoint. It is now immediate that $S / K$ is irreducibly connected between a zero and a unit.

Proof of (5). We show now that $S$ is commutative. By a result of Faucett [5, Lemma 5], $S / K$ is commutative. If $K$ consists of a single element, then $S=S / K$, so that $S$ is commutative. Now suppose that $K$ is not a single element and we will show that $S \backslash K$ is a mob. If not, then for some $x, y \in S \backslash K$ we have $x y \in K$. Note that by Part (3) of the theorem, $K$ is a $C$-set, and hence contains no inner point. Let $[y, u]$ denote the "segment" from $y$ to the unit. Then $x \cdot[y, u]$ is a locally connected continuum which meets $K$ and contains $x$, so $K \subset x \cdot[y, u]$. But since $K$ is a nondegenerate $C$-set, $x \cdot[y, u]$ is not locally connected at any element of $K$, a contradiction. Hence $S \backslash K$ is a mob, and is commutative since $S / K$ is. We conclude that $(S \backslash K)^{*}=S$, so that $S$ is commutative.

Corollary 1. If $S$ is a metric indecomposable continuum with $S^{2}=S$, then either $S$ is a group or the multiplication is of type (i) or (ii).

Proof. If $K$ is proper, then $S$ is irreducible between $K$ and some point not in $K$, so that by the theorem, $S$ has a unit. Hence by [9, Theorem 3$] S$ is a group and $S=K$, a contradiction. We now have $S=K$ and the result follows from the corollary to Theorem 1 .

The following example indicates the complexities that may occur even under what may seem to be the strong hypotheses of the theorem. Let $G$ be a compact group which contains a dense one-parameter semigroup $f\left(R_{+}\right)$ (here $R_{+}$denotes the non-negative additive reals). Define $g: R_{+} \rightarrow R$ by $g(t)$ $=\exp (-t)$. If $h=f \times g$ then $h$ takes $R_{+}$isomorphically into $G \times(0,1)$ and $h\left(R_{+}\right)^{*}$ is a clan whose minimal ideal is the group $G \times\{0\}$ and which is irreducible from $G \times\{0\}$ to $(e, 1), e$ the unit of $G$. We may describe $h\left(R_{+}\right)^{*}$ by saying it is the group $G$ and an infinite half ray spiraling down upon it.

\section{REFERENCES}

1. A. H. Clifford, Semigroups containing minimal ideals, Amer. J. Math. vol. 70 (1948) pp. 521-526.

2. Haskell Cohen, A cohomological definition of dimension for locally compact Hausdorff spaces, Duke Math. J. vol. 21 (1954) pp. 209-224.

3. Eilenberg and Steenrod, Foundations of algebraic topology, Princeton, 1952.

4. W. M. Faucett, Topological semigroups and continua with cutpoints, Proc. Amer. Math. Soc. vol. 6 (1955) pp. 748-756.

5. - Compact semigroups irreducibly connected between two idempotents, Proc. Amer. Math. Soc. vol. 6 (1955) pp. 741-747.

6. W. M. Faucett, R. J. Koch, and K. Numakura, Complements of maximal ideals in compact semigroups, Duke Math. J. vol. 22 (1955) pp. 655-661. 
7. F. B. Jones, Concerning non-aposyndetic continua, Amer. J. Math. vol. 70 (1948) pp. 403-413.

8. R. J. Koch, On monothetic semigroups, Proc. Amer. Math. Soc. vol. 8 (1957) pp. 397401.

9. R. J. Koch and A. D. Wallace, Maximal ideals in compact semigroups, Duke Math. J. vol. 21 (1954) pp. 681-685.

10. K. Kuratowski, Topologie II, Warsaw, 1950.

11. R. L. Moore, Foundations of point set theory, New York, 1932.

12. P. S. Mostert and A. L. Shields, One parameter semigroups in a semigroup (to appear).

13. D. Rees, On semigroups, Proc. Cambridge Philos. Soc. vol. 36 (1940) pp. 387-400.

14. A. D. Wallace, The structure of topological semigroups, Bull. Amer. Math. Soc. vol. 61 (1955) pp. 95-112.

15. - The position of $C$-sets in semigroups, Proc. Amer. Math. Soc. vol. 6 (1955) pp. 639-642.

16. - Cohomology, dimension, and mobs, Summa Brasiliensis Mathematicae vol. 3 (1953) pp. 43-55.

17. - One dimensional homogeneous clans are groups, Indagationes Math. vol. 17 (1955) pp. 578-580. 23-28.

18. - Inverses in Euclidean mobs, Math. J. Okayama University vol. 3 (1953) pp.

19. - The Rees-Suschkewitsch theorem for compact simple semigroups, Proc. Nat. Acad. Sci. U.S.A. vol. 42 (1956) pp. 430-432.

20. - Retraction in semigroups, Pacific J. Math (to appear).

21. L. E. Ward, Jr., Partially ordered topological spaces, Proc. Amer. Math. Soc. vol. 5 (1954) pp. 144-161.

Louisiana State University,

Baton Rouge, La.

TUlane UNIVERSITY,

New Orleans, La. 